\title{
Correlations between Emotion Regulation Behaviors and Processes of Accepting Sports Injuries: From the Perspective of Enacted Social Support
}

\author{
Tomonori Tatsumi ${ }^{1}$, Takashi Takenouchi ${ }^{2}$ \\ ${ }^{1}$ Kio University, Nara, Japan \\ ${ }^{2}$ Nagoya University, Nagoya, Japan \\ Email: t.tatsumi@kio.ac.jp
}

How to cite this paper: Tatsumi, T., \& Takenouchi, T. (2017). Correlations between Emotion Regulation Behaviors and Processes of Accepting Sports Injuries: From the Perspective of Enacted Social Support. Psychology, 8, 1091-1109.

https://doi.org/10.4236/psych.2017.88071

Received: April 25, 2017

Accepted: June 9, 2017

Published: June 12, 2017

Copyright $\odot 2017$ by authors and Scientific Research Publishing Inc. This work is licensed under the Creative Commons Attribution International License (CC BY 4.0).

http://creativecommons.org/licenses/by/4.0/

\begin{abstract}
This study examined the relationships between emotion regulation behavior (ERB) and athletic injury psychological acceptance (AIPA) process which consists of psychosocial recovery factor (PSRF) and AIPA, from the viewpoint of enacted social support (SS). Athletes $(N=180)$ that had experienced an injury were divided into high-and low-SS groups based on SS scale. Next, a model, in which ERB variables have an influence on AIPA process, was examined using multiple-group structural equation modelling. The model indicated differences in the degree of SS. It was suggested that in the high-SS group, ERB of emotional expression and positive reappraisal were functional, and these ERB were positively mediated by PSRF of emotional stability and temporal perspective, respectively, which promoted AIPA. Whereas, it was suggested that ERB of emotional suppression and positive reappraisal were functional, and these ERB were positively mediated by temporal perspective, which promoted AIPA in the low-SS group. However, emotional suppression was also negatively mediated by emotional stability, which obstructed AIPA. These results are suggested that differences in athletes' support environment should be considered when designing interventions.
\end{abstract}

\section{Keywords}

Emotion Regulation, Athletic Injury Psychological Acceptance Process, Social Support

\section{Introduction}

Sports injuries include external injuries caused by external forces applied once to 
the body and impairments caused by repeated microtrauma. Both of these lower athletic abilities owe to organic and functional impairments. To return to doing sports, treatment for a certain period and athletic rehabilitation are indispensable. Recently, acute rehabilitation aiming at early return has become popular, and injured athletes are expected to start rehabilitation just after getting injured. However, it is not always easy for athletes to accept situational changes caused by the injury and positively participate in rehabilitation (Tatsumi \& Nakagomi, 1999). Negative emotions such as anger, depression, and frustration caused by the injury inhibit rehabilitation behaviors (Uemukai, 1992; Tatsumi, 2003). Time and procedures that are appropriate for each person are necessary for injured athletes to accept reality and conduct future-oriented coping behaviors, such as participating in rehabilitation (Tatsumi \& Nakagomi, 1999).

After getting injured, athletes have a disordered sense of the continuity of psychological time, i.e. one's past, present, and future (Tatsumi, 2014). One of the goals of psychological interventions is to provide support for changing the negative recognition of the past and future perspective into a positive one (Katsumata, 1995). It is considered necessary to investigate psychological dynamism of injured athletes when providing psychological support, in order to facilitate these cognitive changes. Fujii's cognitive theory on the continuity of successive athletic ability is suggestive with regard to this issue. Fujii (2000) named injured athletes' cognition of seeking continuity of athletic ability between the past, i.e. before getting injured, and the future "backward cognition." On the other hand, cognition of recognizing the continuity between the present athletic ability, which has declined because of the injury, and future ability was named "forward cognition.” Fujii (2000) explained psychological dynamisms of injured athletes based on the two types of cognitive patterns regarding the continuity of successive athletic ability described above. Especially, in the early period of rehabilitation, injured athletes tend to stick with their former selves and the surrounding social environment before getting injured, and perceive the future as a continuum of their past career and athletic ability they had developed until being injured (Tatsumi, 2014; Tatsumi, Fukumoto, \& Bai, 2015). In the state of this "backward cognition," athletes feel sorrow for the loss and irritation, more strongly than the pleasure of acquiring their lost functions through rehabilitation (Fujii, 2000). On the other hand, when this is changed to "forward cognition," with which athletes perceive the future based on their present ability, the pleasure of acquiring functions become stronger than the pain of their loss (Fujii, 2000). The task for injured athletes is to change their cognition from backward to forward, i.e. to accept the injury and focus on the present, which is the point of restarting (Fujii, 2000; Tatsumi et al., 2015). Here, thementality ofAthletic Injury Psychological Acceptance (AIPA: Tatsumi, 2013) intervenes (Tatsumi, 2014). Previous studies (McDonald \& Hardy, 1990; Nakagomi \& Uemukai, 1994; Tatsumi \& Nakagomi, 1999) have indicated a positive correlation between AIPA and dedication to rehabilitation. Uemukai (1993), Tatsumi (2013), Tatsumi and Takenouchi (2014) quantitatively demonstrated this correlation. Moreo- 
ver, Tatsumi (2012) indicateda positive correlation between AIPA during rehabilitation and cognition of recovery of athletic movements, as well as the sense of self-development when returning to sports. Furthermore, Tatsumi (2014) indicated the level of AIPA during rehabilitation and dedication to rehabilitation affected the state of psychological adaptation after returning to sports. The series of studies cited above have demonstrated the effectiveness of psychological support aiming at AIPA. Tatsumi and Takenouchi (2014) previously examined Psycho-Social Recovery Factors (PSRF) facilitating AIPA. The results of analyses using structural equation modeling indicated that PSRF is related to "emotional stability" and especially the "time perspective" facilitated AIPA. The process consisting these two factors was named as the AIPA process. The effect of emotional stability on AIPA was particularly stronger. The above results suggest that it is necessary to conduct an investigation by adding individual and situational variables that regulate recovery of emotional stability. Therefore, this study regarded Emotional Regulation Behavior (ERB) used by injured athletes to deal with negative emotions as an individual variable and Social Support (SS) provided by important others as a situational variable.

Certain previous studies have investigated emotional regulation of breast cancer patients as a variable affecting psychological responses after the disease (Iwamitsu, Shimoda, Abe, Tani, Kodama, \& Okawa, 2003; Nakatani, Iwamitsu, Kuranami, Okazaki, Yamamoto, Watanabe, Miyaoka, 2012; Stanton, Danoff-Burg, Cameron, Bishop, Collins, Kirk, Sworowski, \& Twillman, 2000). A series of these studies examined correlations between individual emotional suppression tendencies and the level of psychological pain, as well as emotional confusion after a definitive diagnosis, from the perspective of emotional suppression and emotional expression. Moreover, interviews conducted with injured athletes by Mankad, Gordon, and Wallman (2009a) indicated that the participants commonly "adopted evasive behaviors for maintaining group norms" and "suppressed negative feelings for fear of negative evaluation." An intervention study conducted later by Mankad and Gordon (2010) as well as Mankad, Gordon, and Wallman (2009b, 2009c) examined the effectiveness of emotional disclosure through writing therapy. Studies described above suggest the efficacy of studying the injured the ERB of athletes from the perspective of emotional expression and emotional suppression. Furthermore, Gross and John (2003) regarded emotional regulation conducted after emotion generation as Response-focused Emotion Regulation, whereas regulation conducted before emotion generation was regarded as Antecedent-focused Emotion Regulation. They regarded suppression as a typical strategy of the former type of emotional regulation, whereas reappraisal, which is a positive reinterpretation of events, was regarded as a strategy in the latter type of emotional regulation. Based on above studies, this study investigated emotional expression, emotional suppression, and positive reappraisal as ERB.

Cohen, Kessler, and Gordon (1995) indicated the necessity of considering SS provided by a third party as a moderator variable between life events and health 
problems. Injured athletes are in a state in which past SS environment (support networks) are missing. They tend to lose interpersonal communication (Nakagomi \& Uemukai, 1994) and have a strong sense of alienation and isolation (Tatsumi \& Nakagomi, 1999). People in such a state are also considered to have a strong desire for interpersonal relationships and SS (Suzuki \& Nakagomi, 2013). Injured athletes have to deal with psychological pain and rebuild the SS environment to return to sports. When SS is provided by important others, the injured athletes have confidence in receiving SS, which might work effectively in searching for measures to make a breakthrough in the situation (Suzuki \& Nakagomi, 2015).

Moreover, Kennedy-Moore and Watson (2001) considered interpersonal and cognitive processes important as a premise for the benefit produced by expressing psychological pain. Important others have a significant meaning for injured athletes in controlling negative emotions. For example, Kimura (2004) indicated it was effective to reduce the intention of suppression, suggesting "positive replacement thought" as an effective method, based on the Ironic Processes Theory (Wegner, 1994), which advocates paradoxical effects of suppressing undesirable thought. By recalling encouragements of friends and seniors during unpleasant experiences, people might get a new viewpoint and think of new approaches and get encouraged (Kimura, 2004). Furthermore, Fujii (2000) suggested that expression of psychological pain might make it possible to change cognitions from backward to forward, indicating the necessity of time and space (i.e. environment) where people can express their own emotions. In other words, in an environment in which SS provided by important others is sufficiently recognized, intention to suppress psychological pain caused by the injury decreases, and ERB such as emotional expression and positive reappraisal might facilitate AIPA processes. On the other hand, in an environment in which SS provided by important others is recognized insufficiently, suppression intention might not be reduced and emotional suppression might inhibit AIPA processes. Based on the above, when comprehensively perceiving the rehabilitation period, the degree of recognition of SS provided by important others is considered to function as a variable regulating injured athletes' ERB and AIPA processes.

This study examined causal relationships of ERB and AIPA processes (ERB $\rightarrow$ PSRF $\rightarrow$ AIPA) depending on differences in the degree of recognition of SS provided by important others, in active athletes that had been injured university entrance.

\section{Methods}

\subsection{Participants and Procedures}

It was predicted that severity of injuries, the stage of rehabilitation, and injured athletes' dedication to sports would affect mental states of athletes. Therefore, criteria of participants were set as follows: 1) active athletes that had to stop doing sports at least one week because of injury after entering university and conducted rehabilitation, 2) athletes that are not doing rehabilitation at present, 
3) athletes that at least one week had passed after returning to sport, 4) student athletes belonging to university athletic clubs with high competitive levels. Ex-injured athletes that met the four criteria above were regarded as participants in this study. The reason why we regarded injured athletes that had to stop doing sports at least one week as participants is that an early study (Uemukai, 1993) that adopted this criterion confirmed grief and acceptance. Moreover, other previous studies conducted by Tatsumi $(2013,2014)$ as well as Tatsumi and Takenouchi (2014) used the same criterion. Therefore, this study also adopted it.

First, the purpose and outline of this study were explained to the researchers of psychology or rehabilitation at two universities having faculties of physical education and at one university having classes consisting of students that entered the university on sports recommendation, and to advisors to five major athletic clubs at the three universities. Their consent for the study was obtained, including distribution and collection of questionnaires. The questionnaire distributed to the participants was put in an envelope $(240 \mathrm{~mm} \times 332 \mathrm{~mm})$, with a letter indicating the purpose, outline, procedures, request and consent for the survey. Participants were required to take out the questionnaire from the envelope by themselves and respond anonymously. After responding, they were required to put it in the envelope and seal it using adhesive tape prepared in the flap. Subsequently, they were required to put the envelope in a larger envelope $(270 \mathrm{~mm} \times 382 \mathrm{~mm})$ by themselves. Through adopting the procedure above, anonymity of the participants was supposed to be maintained. We also instructed those that did not agree with the survey to put the unanswered questionnaire in the envelope following the same procedure. However, there were no persons corresponding to that instruction.

The questionnaire was distributed to 200 persons and all of them were collected. The number of valid responses, excluding those that did not met the criteria of the participants and those with missing data, was 180 (70 men, 110 women, mean age $=20.27, S D=1.02)$. The events that participants played varied: the number of soccer players was 32 (17.78\%), the number of basketball players was $31(17.22 \%)$, the number of track and field players was 19 (10.56\%), the number of judo players was $19(10.56 \%)$, the number of handball players was $16(8.89 \%)$, the number of soft tennis players was $12(6.67 \%)$, the number of kendo players was $12(6.67 \%)$, the number of softball players was 10 (5.56\%), the number of wrestling players was 9 (5.00\%), and the number of 12 other events players was $20(11.11 \%)$. The competitive levels of the teams participants belonged to were rather high: the number of participants belonging to the teams with the higher level than the fourth ranking in Japan or the level of participating in international competitions was 55 (30.56\%), the number of those belonging to the teams with the level of the fifth-eighth ranking in Japan was 56 $(31.11 \%)$, the number of those belonging to the teams with the level of the ninth ranking in Japan or the level of participating in national competitions was 68 $(37.78 \%)$, and the number of those belonging to the team ranked higher in a local league was one $(0.56 \%)$. All the injuries reported by the participants were or- 
thopedic injuries: the number of participants who reported muscle ligament tear or rupture was 155 (86.11\%), the number of those who reported fracture was 20 (11.11\%), and the number of those who reported joint dislocation was 5 (2.78\%). 10 participants did not have a record of the number of days of stopping sports because of injuries. However, we judged that they were the injuries requiring at least one-week stop, based on the diagnosis and the day when they got injured as well as the day they returned (when they participated in general club activities). Excluding these 10 participants, the average number of days during which participants were stopped doing sports was 66.58 days $(S D=87.13)$. The survey was executed from November to December in 2014, after deliberation at the ethics committee of Kio University.

\subsection{Measures}

The following scales were used in measurement: a scale for assessing SS provided by others during rehabilitation, the emotional stability scale and time perspective scale related to ERB and PSRF used for negative emotions, and scales related to AIPA.

Social Support. For developing the Athletic Injury Social Support Scale (AISS-S), free description was conducted with ex-injured participants $(N=87$, 69 men, 18 women, mean age $=20.26, S D=1.10$ ) different from the participants in this survey about SS they required during rehabilitation. As a result, 50 concrete descriptions were collected. Through examination using the KJ method, these descriptions were classified into three types of SS and labels were attached. The preliminary version of AISS-S was developed based on the results above, including 28 items as follows: "listening, empathy, and acceptance (9 items)," "social approval and acceptance (10 items)," and "offer of advice about rehabilitation and information provision (9 items)." In the process of developing AISS-S, content validity was examined by athletic rehabilitation specialists. When three among five specialists judged as valid, the item was adopted. Consequently, all the items were judged as valid.

Emotion Regulation Behavior. "Positive reappraisal (4 items)" and "emotional expression (4 items)" developed by Nozaki (2013) were used. Moreover, referring to the Emotional Suppression Tendency Scale developed by Kashimura and Iwamitsu (2007), four items of "emotional suppression" based on the context of injuries were added, and the provisional version of the Emotion Regulation Behavior Scale (ERB-S) consisting of 12 items was developed.

Psycho-Social Recovery Factors. The degree of recovery of emotional stability and time perspective was measured using the Emotional Stability scale and Time Perspective scale, sub-scales of the Psycho-Social Recovery Factor Scale (PSRF-S) developed by Tatsumi and Takenouchi (2014). We adopted four items that showed higher factor loadings from each scale, in total eight items. Reliability of the emotional stability scale and time perspective scale was confirmed using Cronbach's alpha, indicating .80 and .78 respectively, which were sufficiently reliable. 
Athletic Injury Psychological Acceptance. The degree of AIPA was measured using seven items of the Athletic Injury Psychological Acceptance Scale (AIPA-S) developed by Tatsumi (2013). Cronbach's alpha was confirmed and it was .77, which was sufficiently reliable.

Participants were required to reflect their whole life during rehabilitation. Regarding AISS-S, the degree of recognition of SS provided during rehabilitation was inquired using the four-point scale (1: Not at all 4: Often) ${ }^{1}$. Regarding four items of emotional suppression of ERB-S and PSRF-S as well as AIPA-S, the state during rehabilitation was inquired using the seven-point scale (1: Never applicable 7: Very applicable). Eight items of emotional expression and emotional suppression of ERB-S were inquired using the five-point scale (1: Never applicable 5: Very applicable). Moreover, participants were required to describe the injury name, the number of days of stopping sports based on the doctor's diagnosis, athletic events they participated in, and competition results after entering university. Those having got injured several times after entering university were required to reflect one experience that was most impressive.

\subsection{Data Analysis}

First, factor analysis was conducted on 28 items of the preliminary version of AISS-S and 12 items of the preliminary version of ERB-S, and factorial validity of the scales was examined. Reliability of the scales was examined using Cronbach's alpha. Next, based on the mean value of AISS-S, participants were classified into the high SS group and low SS group. Differences in the mean value of each scale between two groups were examined using a $t$-test and the effect size $(r)$ was calculated. The scale scores of sub-scales of AISS-S and ERB-S, the emotional stability scale and time perspective scale related to PSRF-S, and AIPA-S were calculated through adding all the scores of each scale and dividing it by the number of the items. Subsequently, the level of correlations among scales was examined using Pearson's product-moment correlation coefficient, depending on the group (high SS and low SS), and an early causal model, in which ERB affects AIPA mediated by two variables of PSRF, was developed. Finally, goodness-of-fit of the model was examined depending on the group, using covariance structure analysis, and path coefficients between groups were compared using multi-group analysis. In multi-group analysis, aiming to confirm configural invariance, at first a model without imposing equality constraints between groups (configural invariance model) was examined. Next, the model was compared with other models with imposing equality constraints. Whether indirect effects were significant or not was examined using the Distribution of the product method (Tofighi \& MacKinnon, 2011), through calculating 95\% confidence intervals (CIs) of the product of random variables.

\section{Results}

\subsection{Factor Analysis}

First, Exploratory factor analysis (principal factor method, promax rotation) was 
conducted on 28 items of the preliminary version of AISS-S. Items showing the absolute value of factor loadings less than .40 and items showing high factor loadings to plural items were excluded successively, and factor analysis was repeatedly conducted. As a result, three factors with the eigenvalue of over 1.0 were extracted (Table 1). The first factor included seven items related to listening to, sympathy with, and acceptance of their worries and feelings, and the factor was interpreted as a "listening, sympathy, and acceptance" factor. The second factor included seven items relevant to offering assessment, advice, and information about rehabilitation. This factor was interpreted as a factor "offer of advice about rehabilitation and information provision." The third factor included eight items related to encouragement for themselves that had to leave sports temporarily, and helping them become aware of their significance. This factor was interpreted as a "social approval and acceptance" factor. The fit indices of the three factor solution model by Confirmatory factor analysis were SRMR $=.05$, RMSEA $=.09, \mathrm{CFI}=.90, \mathrm{AIC}=622.38$. Furthermore, inter-factor correlations were in the range of $r=.69-.73$. Such strong correlations among factors suggest the need that should examine the one factor solution model or two factor solution model. Therefore we examined the models that adopted one factor solution and two factor solution by Confirmatory factor analysis. As a result, the fit indices of the one factor solution model were SRMR $=.06$, RMSEA $=.12$, CFI=

Table 1. Factor loadings for Exploratory factor analysis with promaxrotation of athletic injury social support scale $(N=180)$.

\begin{tabular}{|c|c|c|c|c|}
\hline & & $\mathrm{F} 1$ & $\mathrm{~F} 2$ & F3 \\
\hline \multicolumn{5}{|l|}{ Factor 1: Listening, Sympathy, and acceptance $(\alpha=.94)$} \\
\hline Sympathizing with my feelings by being injuries & & .87 & -.01 & -.04 \\
\hline Understanding worries and pains caused by injuries & & .86 & .20 & -.14 \\
\hline Listening to worries and pains caused by injuries & & .73 & -.07 & .23 \\
\hline Encouraging me when I am depressed & & .70 & -.04 & .17 \\
\hline Listening to my worries and pains caused by being injured & & .60 & .07 & .24 \\
\hline Considering worries and anxieties & & .54 & .15 & .24 \\
\hline Providing consultations on worries and pains caused by injuries & & .53 & .17 & .16 \\
\hline \multicolumn{5}{|l|}{ Factor 2: Offer of Advice about Rehabilitation and Information Provision $(\alpha=.91)$} \\
\hline Making a regular assessment of the effects of rehabilitation & & -.03 & .75 & .10 \\
\hline Concretely indicating causes of the injury and how to comeback & & .25 & .73 & -.07 \\
\hline Providing consultation for coming back, such as making rehabilitation plans & & .10 & .72 & .02 \\
\hline Giving information about hospitals and rehabilitation facilities depending on the injury & & -.02 & .68 & .05 \\
\hline Giving information useful for coming back, such as rehabilitation methods & & .01 & .68 & .09 \\
\hline Concretely indicating the state of the injury and treatments & & .36 & .66 & -.19 \\
\hline Giving advice for recovery and comeback & & -.09 & .65 & .25 \\
\hline \multicolumn{5}{|l|}{ Factor 3: Social Approval and Acceptance $(\alpha=.92)$} \\
\hline Supporting me who was injured & & .14 & .00 & .74 \\
\hline Making me feel the significance and necessity of having rehabilitation & & -.32 & .44 & .68 \\
\hline Sending messages saying that I am a teammate playing together & & .33 & -.07 & .62 \\
\hline Offering words that make me motivated & & .19 & .12 & .55 \\
\hline Considering me in a casual manner & & .39 & -.09 & .55 \\
\hline Talking to me as usual even when I am injured & & .01 & .02 & .54 \\
\hline Sending messages saying that I am necessary for the team & & .32 & -.02 & .53 \\
\hline Thinking, so that I can easily go to the practice place or game venues & & .18 & .18 & .47 \\
\hline \multirow[t]{2}{*}{ Correlation coefficient } & F1 & - & .70 & .73 \\
\hline & $\mathrm{F} 2$ & - & - & .69 \\
\hline
\end{tabular}


$.82, \mathrm{AIC}=871.34$, and the fit indices of two factor solution model were SRMR $=.05, \mathrm{RMSEA}=.10, \mathrm{CFI}=.88, \mathrm{AIC}=.681 .27$. The above-mentioned results suggested that the three factor solution model was the best.

Ura (1992) focused on functions of SS, and classified SS into two types, i.e. SS directly or indirectly works in solving problems and SS works on the cognitive and emotional aspects. From this perspective, "offer of advice about rehabilitation and information provision" is considered to correspond to the former SS type and "listening, sympathy, and acceptance" as well as "social approval and acceptance" are considered to correspond to the latter SS type. AISS-S includes two types of SS functions above, and it is considered valid conceptually. Among the extracted three factors, the "listening, sympathy, and acceptance" factor corresponds to emotional support, which is the sub-scale of the Social Support Inventory for Injured Athletes (SSIIA) developed by Mitchell, Rees, Evans, and Hardy (2005) and the Athletes' Received Support Questionnaire (ARSQ) developed by Freeman, Coffee, Moll, Rees, and Sammy (2014). On the other hand, the "social approval and acceptance" factor corresponds to esteem support, and the "offer of advice about rehabilitation and information provision" factor corresponds to information support as well as tangible support. Based on the above, invariance of the scales developed through previous studies and AISS-S factors was suggested, and factorial validity of AISS-S was considered to be ensured. The alpha coefficient of the whole scale was .97, alpha coefficients of sub-scales were in the range of .91 - .94, indicating sufficient internal consistency of the whole scale and sub-scales. Furthermore, strong correlations among factors mentioned above suggest that injured athletes generally recognize originality of various SS provided by others, and receive various SS in a balanced manner, when they have recognized that SS had been enacted. Such strong correlations among factors were confirmed in the study conducted by Rees, Mitchell, Evans and Hardy (2010) using SSIIA (Mitchell et al., 2005). They adopted analysis procedures using composite scores of the sub-scales by the reason of the correlations between the social support dimensions. In the present study, based on the procedures used by Rees et al. (2010), composite scores of three sub-scales were used in the following analyses.

Next, Exploratory factor analysis (principal factor method, promax rotation) was conducted on 12 items of the preliminary version of ERB-S. As a result, three factors having an eigenvalue of over 1.0 were extracted (Table 2). As assumed in the stage of constructing scales, the first factor consisted of four items related to emotional suppression, the second factor consisted of four items related to positive reappraisal, and the third factor consisted of four items related to emotional expression. The fit indices of the three factor solution model by Confirmatory factor analysis were SRMR $=.07, \mathrm{RMSEA}=.08, \mathrm{CFI}=.92$ Based on the above, factorial validity of the scale was confirmed. The alpha coefficients of the three factors were $.82, .81$, and .75 respectively, and reliability of each scale was ensured. Though a negative correlation $(r=-.17)$ was indicated between factors of emotional suppression and emotional expression, the value was rather 
Table 2. Factor loadings for exploratory factor analysis with promaxrotation of emotion regulation behavior scale $(N=180)$.

\begin{tabular}{|c|c|c|c|c|}
\hline & & $\mathrm{F} 1$ & F2 & F3 \\
\hline \multicolumn{5}{|l|}{ Factor 1: Emotional Suppression $(\alpha=.82)$} \\
\hline \multicolumn{5}{|l|}{ I hid my negative feelings at all costs } \\
\hline \multicolumn{2}{|l|}{ I tried to control negative feelings by myself } & .93 & -.01 & .04 \\
\hline \multicolumn{2}{|l|}{ I tried to keep negative feelings to myself } & .83 & .02 & -.01 \\
\hline \multicolumn{2}{|l|}{ I did not tell my negative feelings to others } & .66 & -.03 & .03 \\
\hline \multicolumn{2}{|l|}{ Factor 2: Positive Reappraisal $(\alpha=.81)$} & .50 & .05 & -.14 \\
\hline \multicolumn{5}{|l|}{$\begin{array}{l}\text { Factor 2: Positive Reappraisal }(\alpha=.81) \\
\text { I tried to learn something from experiences in order to have positive feelings }\end{array}$} \\
\hline \multirow{2}{*}{\multicolumn{2}{|c|}{$\begin{array}{l}\text { In order to relieve negative feelings, I tried to think that the experience would be beneficial to me when I get } \\
\text { over it }\end{array}$}} & .06 & .83 & -.07 \\
\hline & & -.10 & .82 & -.14 \\
\hline \multicolumn{2}{|l|}{ In order to have positive feelings, I tried to look for good aspects of things that were happening to me } & .04 & .67 & .13 \\
\hline \multicolumn{2}{|l|}{ In order to relieve negative feelings, I tried to regard the incident as a good experience } & .04 & .57 & .15 \\
\hline \multicolumn{5}{|l|}{ Factor 3: Emotional Expression $(\alpha=.75)$} \\
\hline \multicolumn{2}{|l|}{ In order to have positive feelings, I did something to let my negative feeling out } & -.03 & .00 & .69 \\
\hline \multicolumn{2}{|l|}{ In order to relieve negative feelings, I tried not to suppress negative feelings inside myself } & .03 & .02 & .69 \\
\hline \multicolumn{2}{|l|}{ In order to relieve negative feelings, I did something to show my negative feelings } & .00 & -.14 & .68 \\
\hline \multicolumn{2}{|l|}{ In order to have positive feelings, I tried not to bottle up negative feelings inside myself } & -.08 & .22 & .55 \\
\hline Correlation coefficient & F1 & - & .28 & -.17 \\
\hline Correlation coemicient & $\begin{array}{l}\text { F1 } \\
\text { F2 }\end{array}$ & - & - & .18 \\
\hline
\end{tabular}

small. The results above suggest that both variables cannot be evaluated in the same dimension. That is, injured athletes do not recognize behaviors expressing emotions and behaviors not suppressing emotional expressions as ERB having the same meaning. Though studies on emotion regulation in daily life (Gross \& John, 2003; Yoshizu, Sekiguchi, \& Amamiya, 2013) confirmed sex differences in the use of emotion regulation strategies, the results of this study did not indicate sex differences as follows: emotional suppression (men: $M=4.31, S D=1.43$, women: $M=4.55, S D=1.13$ ), positive reappraisal (men: $M=3.55, S D=.98$, women: $M=3.70, S D=.81$ ), emotional expression (men: $M=2.75, S D=.79$, women: $M=2.83, S D=.80)$, and all of them were not significant $(t=-1.28$, $p>.05 ; t=-1.15, p>.05 ; t=-.65, p>.05$, respectively).

Furthermore, the number of days athletes had to stop doing sports was regarded as the index of severity of injuries. A correlation between the number of days and AISS was $r=.07$ in the whole analysis subjects $(N=170)$, and correlations between the number of days and three variables related to ERB were rather low $(r=.00-.13)$ and all of them were not significant $(p>.05)$.

Lastly, correlations between AISS-S composite scores and ERB-S were as follows: emotional suppression $(r=.16, p<.05)$, positive reappraisal $(r=.27, p$ $<.01)$, and emotional expression $(r=.20, p<.01)$, which were rather small, suggesting it is possible to distinguish construct of SS from that of ERB. Subsequently, causal relationships between ERB and AIPA processes were examined from the perspective of the differences in the degree of recognition of SS provided by important others.

\subsection{Relationships between ERB and AIPA Processes Based on the Difference in the Degree of Recognition to Enacted SS}

Based on the examination made in 3.1., participants were classified into the high 
SS group $(M=3.46, S D=.35)$ and low SS group $(M=2.39, S D=.47)$, with setting the mean value (2.93) of composite scores of recognition to SS enacted during rehabilitation as a criterion. The result of a $t$-test indicated that the difference in the SS composite scores between two groups was significant $(t(178)=17.37$, $p<.001, r=.79$ ). Subsequently, the mean values of high and low SS groups were examined using a $t$-test, regarding six scales used in the following analyses. The results are shown in Table 3. Significant differences were indicated in the mean scores of positive reappraisal $(t(169.05)=2.92, p<.01, r=.22)$ and emotional expression $(t(178)=2.09, p<.05, r=.16)$ of ERB-S, as well as time perspective $(t(178)=4.21, p<.001, r=.30)$ of PSRF-S. All the scores of the high SS group were higher than low SS group. On the other hand, regarding the mean value of emotional stability $(t(178)=-1.76, p<.10, r=.13)$, the low SS group indicated a higher score. Moreover, the differences in the mean values of two groups regarding emotional suppression and AIPA were not significant $(t(178)=1.42, p$ $=.16 ; t(178)=1.40, p=.17$, respectively). That is, high SS group used ERB related to positive reappraisal and emotional expression more often than low SS group, and the recovery level of time perspective was higher. On the other hand, low SS group did not use both ERB as often as high SS group, the recovery level of time perspective was lower, whereas the recovery level of emotional stability was maintained higher.

Correlation coefficients among scales in high and low SS groups are shown in Table 4. Correlations between positive reappraisal and AIPA, emotional stability and AIPA, and time perspective and AIPA were significant in both high and low SS groups: in the high SS group, $r=.36-.52$, a correlation between positive reappraisal and AIPA was $p<.01$, and correlations between other factors and AIPA were $p<.001$. In the low SS group, $r=.40-.61$, correlations between all the factors and AIPA was $p<.001$. Moreover, a correlation between positive reappraisal and time perspective was significant (high SS group: $r=.29, p<.01$; low SS group: $r=.54, p<.001)$. On the other hand, a correlation between emotional expression and emotional stability was significant in the high SS group $(r$ $=.31, p<.01)$, whereas it was not significant in the low SS group $(r=.02, p=.86)$. Though correlations between emotional suppression and emotional stability as

Table 3. Descriptive statistics andt-test result of the major study variables in high and low-SS group.

\begin{tabular}{lcccccccc}
\hline & \multicolumn{1}{c}{ High SS $(n=91)$} & \multicolumn{2}{l}{ Low SS $(n=89)$} \\
& $M$ & $S D$ & $M$ & $S D$ & $t$ & $p$ & $r$ \\
\cline { 2 - 9 } & & & & & & & \\
& & & & & & & & \\
1. Emotional Suppression & 4.59 & 1.21 & 4.32 & 1.29 & 1.42 & & .11 \\
2. Positive Reappraisal & 3.83 & 0.77 & 3.46 & 0.95 & 2.92 & $* *$ & .22 \\
3. Emotional Expression & 2.92 & 0.79 & 2.67 & 0.79 & 2.09 & $\star$ & .16 \\
4. Emotional Stability & 3.50 & 1.28 & 3.88 & 1.55 & -1.76 & $\dagger$ & .13 \\
5. Time Perspective & 5.41 & 0.93 & 4.76 & 1.15 & 4.21 & $* * *$ & .30 \\
6. AIPA & 4.55 & 1.00 & 4.33 & 1.10 & 1.40 & & .11 \\
& & & & & & & \\
\hline
\end{tabular}

$\dagger p<.10,{ }^{*} p<.05,{ }^{* *} p<.01,{ }^{* * *} p<.001$. 
Table 4. Correlation analysis result of the major study variables in high and low-SS group.

\begin{tabular}{lcccccc}
\hline & \multicolumn{7}{c}{ Correlation coefficient $(r)$} \\
\cline { 2 - 7 } & 1 & 2 & 3 & 4 & 5 & 6 \\
\hline 1. Emotional Suppression & - & .09 & -.13 & .06 & .16 & .03 \\
2. Positive Reappraisal & $.33^{* *}$ & - & $.30^{* *}$ & $.19 \dagger$ & $.29^{* *}$ & $.36^{* *}$ \\
3. Emotional Expression & $-.24^{*}$ & .04 & - & $.31^{* *}$ & -.05 & .02 \\
4. Emotional Stability & $-.36^{* *}$ & .03 & .02 & - & .12 & $.37^{* * *}$ \\
5. Time Perspective & $.45^{* * *}$ & $.54^{* * *}$ & .00 & -.08 & - & $.52^{* * *}$ \\
6. AIPA & .12 & $.46^{* *}$ & -.05 & $.40^{* * *}$ & $.61^{* * *}$ & - \\
\hline
\end{tabular}

$\dagger p<.10,{ }^{*} p<.05,{ }^{* *} p<.01,{ }^{* * *} p<.001$. Note: The upper section of the diagonal of the right column is a coefficient of high-SS group $(n=91)$, and the lower section is a coefficient of low-SS group $(n=89)$.

well as time perspective were not significant in the high SS group $(r=.06, p$ $=.56 ; r=.16, p=.12$, respectively), they were significant in the low SS group ( $r$ $=-.36, p<.01 ; r=.45, p<.001$, respectively). The results above suggest that positive reappraisal and emotional expression might affect AIPA processes in the high SS group, whereas positive reappraisal and emotional suppression might affect AIPA processes in the low SS group.

Based on the results of correlation analysis above, an early causal model was developed and examined through covariance structure analysis. First, considering the result that a correlation of either high or low SS group was significant, covariance was assumed among the three variables of ERB, which were exogenous variables. Next, goodness-of-fit indices of the model were calculated regarding the high and low SS groups respectively. The early causal model was developed using paths with significant correlation coefficients in both groups or in either of them. As a result, goodness-of-fit indices in the high SS group were $\chi^{2}$ $(5)=6.59(p=.25)$, SRMR $=.05, \mathrm{RMSEA}=.06, \mathrm{CFI}=.98$, and that in the low SS group were $\chi^{2}(5)=4.90(p=.43)$, SRMR $=.04$, RMSEA $=.00$, CFI $=1.00$, and both of them were considered to be sufficient. Subsequently, aiming to confirm configural invariance between high and low SS groups, goodness of fit of the model without imposing equality constraints on every path between high-low SS groups (the configural invariance model) was examined through multi-group analysis. Obtained goodness-of-fit indices were $\chi^{2}(10)=11.48(p=.32)$, SRMR $=$ .05 , RMSEA $=.03$, CFI $=.99$, and they were sufficient. The results above indicated the structure of the analysis model was assumed to be equal between high and low SS groups. Next, differences in the estimate values of path coefficients between high and low SS groups were examined. The results indicated $\mathrm{z}$ value of the path coefficient from emotional suppression to emotional stability $(\mathrm{z}=$ $-3.44, p<.001)$ and that from emotional expression to emotional stability $(\mathrm{z}=$ $-2.59, p<.001)$ were significant, and differences among two groups were confirmed. On the other hand, significant $\mathrm{z}$ values were not indicated in the path coefficient from positive reappraisal to time perspective $(\mathrm{z}=1.24, n s)$ as well as to AIPA $(\mathrm{z}=-.55, n s)$, from emotional suppression to time perspective $(\mathrm{z}=$ $1.47, n s)$, from emotional stability to AIPA $(\mathrm{z}=1.16, n s)$, from time Perspective 
to AIPA $(\mathrm{z}=.67, n s)$, from emotional suppression to emotional expression $(\mathrm{z}=$ $-.80, n s)$ as well as to positive reappraisal $(\mathrm{z}=1.89, n s)$, and from positive reappraisal to emotional expression $(\mathrm{z}=-1.46, n s)$. Differences between high-low SS groups were not indicated regarding these paths.

Subsequently, simultaneous analysis was conducted on the three models below. Model 1: the configural invariance model without imposing equality constraints on all the paths between high and low SS groups, Model 2: the constraint model with imposing equality constraints on eight paths that did not indicate significant $\mathrm{z}$ values between groups, and Model 3: the partially constraint model with imposing equality constraints on just four paths (from positive reappraisal to time perspective, from positive reappraisal to AIPA, from emotional stability to AIPA, and from time perspective to AIPA) that indicated significant path coefficients in both high and low SS groups, among the paths that did not indicate significant $\mathrm{z}$ values, and goodness-of-fit indices of the models were compared. Based on RMSEA, CFI, and AIC values, goodness of fit of Model 3 was considered best (Table 5). The results above suggest it is necessary to consider the difference in the degree of enacted SS regarding the following paths: those among three variables of ERB on which equality constraints were not imposed, those from emotional suppression to emotional stability as well as to time perspective, and the path from emotional expression to emotional stability. Figure 1 shows the analysis results of Model 3.

First, paths from ERB to PSRF and AIPA were examined. In the high SS group, emotional expression had a positive effect on emotional stability (.33) and emotional stability had a positive effect on AIPA (.35). 95\% CIs of the product of two path coefficients $(.113, S E=.058)$ were $[.002, .231]$. On the other hand, in the low SS group, emotional suppression had a negative effect on emotional stability (-.37) and emotional stability had a positive effect on AIPA (.42). $95 \%$ CIs of the product of two path coefficients $(.159, S E=.054)$ were $[-.269$, -.055]. Furthermore, in the low SS group, emotional suppression had a positive effect on time perspective (.33) and time perspective had a positive effect on AIPA (.56). 95\% CIs of the product of two path coefficients $(.188, S E=.048)$ were $[.098, .287]$. Positive reappraisal had a positive effect on time perspective, regardless of the SS levels (high SS group: .36; low SS group: .38), and time perspective had a positive effect on AIPA (high SS group: .47; low SS group: .56).

Table 5. Goodness of fit indice in the models.

\begin{tabular}{cccccccc}
\hline & $\chi^{2}$ & $d f$ & $p$-value & SRMR & RMSEA & CFI & AIC \\
\hline Model 1 & 11.48 & 10 & .32 & .05 & .03 & .99 & 75.48 \\
Model 2 & 23.77 & 18 & .16 & .08 & .04 & .97 & 71.77 \\
Model 3 & 14.78 & 14 & .39 & .06 & .02 & 1.00 & 70.78 \\
\hline
\end{tabular}

Note: $\chi^{2}=$ chi-square test; $d f=$ degree of freedom; SRMR = standardized root mean square residual; RMSEA = root-mean-square error of approximation; CFI = comparative fit index; AIC = akaike's information criterion. Model 1 points at a configural invariant model. Model 2 points at the model that limited all the passes that $\mathrm{z}$-value is not significant. Model 3 points at the model that limited only the pass that $\mathrm{z}$-value is not significant and the standardization coefficient of both groups is significant. 


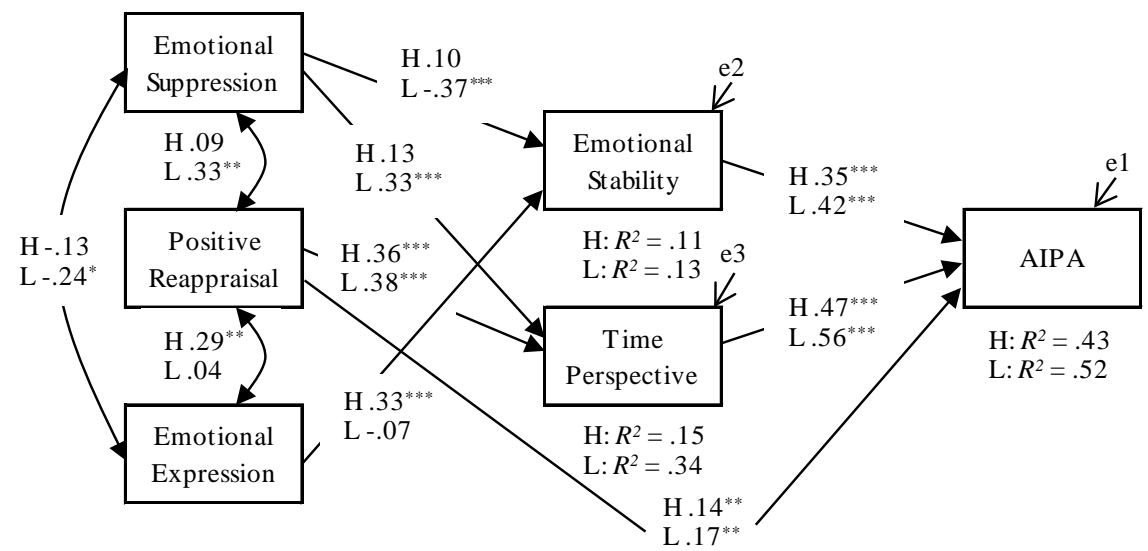

Figure 1. Multiple groups SEM's result concerning a model of causal relationships between ERB and AIPA process $\left(\chi^{2}=14.78 ; d f=14 ; p=.39\right.$; SRMR $=.39$; RMSEA = .02; $\mathrm{CFI}=1.00 ; \mathrm{AIC}=70.78)$. Note: $\mathrm{ERB}=$ emotion regulation behavior; $\mathrm{AIPA}=$ athletic injurypsy chological acceptance; $\mathrm{H}=$ high-social support; $\mathrm{L}=$ low-social support. Numerical values are standardization coefficients.

95\% CIs of the product of two path coefficients in each group (high SS group: $.169, S E=.044$; low SS group: $.211, S E=.051$ ) were [high SS group: .088, .260; low SS group: .117, .316]. Indirect effects indicated above were considered significant because 95\% CLs did not include 0. Moreover, Positive reappraisal had a positive effect on AIPA, not mediated by PSRF, regardless of the SS levels (high SS group: .14; low SS group: .17). Furthermore, paths among variables of ERB, which are exogenous variables, were examined. In the high SS group, a positive correlation (.29) was confirmed between emotional expression and positive reappraisal. In the low SS group, a positive correlation (.33) was indicated between emotional suppression and positive reappraisal, whereas a negative correlation $(-.24)$ was indicated between emotional suppression and emotional expression. 95\% CIs of the indirect effect of emotional suppression on AIPA mediated by emotional stability $(.036, S E=.037)$ in the high SS group were [-.036, $.110]$, and $95 \%$ CLs of the indirect effect of emotional suppression on AIPA mediated by time perspective $(.060, S E=.037)$ in the high SS group were [-.011, $.136] .95 \%$ CIs of the indirect effect of emotional expression on AIPA mediated by emotional stability in the low SS group $(-.030, S E=.085)$ were $[-.198, .137]$. These indirect effects were considered insignificant, because $95 \%$ CIs included 0 .

The results above indicate below: 1) in the high SS group, ERB related to emotional expression has a positive indirect effect; i.e. it facilitates recovery of emotional stability, which promotes AIPA. 2) In the low SS group, ERB related to emotional suppression has a negative indirect effect, i.e. it hinders recovery of emotional stability, which inhibits AIPA. 3) In the low SS group, ERB related to emotional suppression has a positive indirect effect, i.e. it facilitates recovery of time perspective and promotes AIPA. 4) Positive reappraisal works regardless of the level of enacted SS. It has a positive indirect effect on AIPA, i.e. it promotes AIPA mediated by the recovery of time perspective, and also has a positive direct effect on AIPA, i.e. it facilitates AIPA without being mediated by two variables 
related to PSRF. 5) Regarding ERB, emotional expression is positively correlated with positive reappraisal in the high SS group, whereas emotional suppression is positively correlated with positive reappraisal in the low SS group.

\section{Discussion}

This study examined the effects of ERB on AIPA processes from the perspective of differences in the degree of recognition of enacted SS. The results of a $t$-test did not indicate differences in the use of emotional suppression between the high and low SS groups. On the other hand, the high SS group used emotional expression and positive reappraisal more often than the low SS group. That is, though the level of recognition of provided SS did not directly affect the use of emotional suppression, it might have contributed to "reducing intention of suppression," indicated by Kimura (2004), which might have facilitated the use of emotional expression and positive reappraisal. Based on the results of multi-group analysis, injured athletes with high recognition of enacted SS adopted ERB related to emotional expression and positive reappraisal more often than those with low recognition of SS. Moreover, utilized emotional expression and positive reappraisal are considered to have facilitated AIPA directly (positive reappraisal $\rightarrow$ AIPA) or indirectly (emotional expression $\rightarrow$ emotional stability $\rightarrow$ AIPA and positive reappraisal $\rightarrow$ time perspective $\rightarrow$ AIPA). McDonald and Hardy (1990) mentioned acceptance of injuries and indicated the importance of emotional expression, i.e. expression of negative emotions honestly and thoroughly by injured athletes. The results of this study support their findings. Adding to that, the present study suggests the importance of SS environment that would receive negative feelings demonstrated by injured athletes, so that emotional expression would sufficiently function and facilitate AIPA. Three factors of SS were extracted through this study, i.e. 1) listening, sympathy, and acceptance, 2) social approval and acceptance, and 3) offer of advice about rehabilitation and information provision. These three factors were strongly correlated, suggesting the state where injured athletes come to receive various SS, starting from receiving a certain type of SS. For example, when instrumental SS is provided at first, such as offering advice and information about rehabilitation, subsequently emotional involvement would be assumed to be provided. Such diversified SS would support injured athletes' emotional expression, create alternative ideas such as positive reappraisal, and facilitate AIPA.

On the other hand, negative indirect effects were confirmed in the low SS group, i.e. ERB related to emotional suppression hindered recovery of emotional stability and inhibited AIPA. Though examination using a $t$-test did not show differences in the use of emotional suppression between high and low SS groups, the low SS group did not use emotional expression and positive reappraisal as often as the high SS group. That is, though the level of emotional suppression was equal between the two groups, the low SS group did not use emotional expression as often as the high SS group, which accumulated negative feelings, and emotional suppression was considered to have prevented emotional stability 
from recovering and inhibited AIPA. Iwamitsu et al. (2003) and Nakatani et al. (2012) conducted research with breast cancer patients and indicated patients that suppressed their emotions felt psychological pain more strongly than those expressing their emotions. Moreover, Nakatani, Iwamitsu, Yamamoto, and Miyaoka (2014) regarded the feeling of despair as a variable mediating emotional suppression tendencies and psychological pain. Many other studies have demonstrated it is difficult to intentionally suppress negative feelings and the opposite effect is produced in the aspects of thinking and feelings (Gross \& John, 2003; Kimura, 2004; Oikawa \& Oikawa, 2013; Wegner et al., 1993). The results of the present study support the findings of these studies. Based on the above, effectiveness of facilitating expression of negative emotions through providing SS is suggested.

On the other hand, suppression of emotional expression does not always have negative effects. For example, Yoshizu (2014) has indicated whereas emotional suppression has a direct negative effect on self-esteem and subjective happiness, these feelings could be maintained positively through being mediated by selfcontrol skills. Moreover, Kashimura and Iwamitsu (2007) indicated though continuity of emotional suppression has a maladaptive aspect, it is useful for temporally taking a distance from the problem, preventing the problem from getting worse, protecting oneself, and maintaining interpersonal relationships. The present study indicated both emotional suppression and positive reappraisal facilitated recovery of time perspective and promoted AIPA in the low SS group. It is supposed that injured athletes included in the low SS group re-perceived things positively, suppressed emotional expressions, and intended to recover time perspective by themselves through controlling themselves. Considering the results of a $t$-test that differences in the level of AIPA between high and low SS groups were not significant, a series of mechanisms of emotional suppression and positive reappraisal in the low SS group could be interpreted as a kind of adaptation mechanism in injured athletes with low recognition of enacted SS.

However, when such types of injured athletes are placed in the situation where it is difficult for them to have future perspectives by themselves, careful consideration is required. ERB related to emotional suppression hardly gives direct cues to people around the injured athletes for enacting SS. Actually, Gross and John (2003) indicated negative correlations between emotional suppression and SS, close relationships, as well as sharing emotions with others. Furthermore, injured athletes in the low SS group that hardly express their emotions might originally have a tendency of not seeking interactions with others or receiving SS. Especially, when rehabilitation continues for a long time, stagnation and a decline in athletic performance levels and problems related to prognoses might have bad effects on time perspective and emotional aspects. Emotional suppression prevents negative emotions from escaping, and the negative emotions hinder recovery of emotional stability and inhibit AIPA. Such negative effects of ERB have to be noted. Though positive effects of encouraging emotional expression on injured athletes in the low SS group might not be expected, support pro- 
viders should pay attention to the other aspect of this study, i.e. ERB that does not suppress emotional expression might contribute to recovery of emotional stability and promotion of AIPA.

Finally, the results of this study are summarized and its limits and future tasks are described. From the perspective of the level of recognition of enacted SS, differences in ERB adopted by injured athletes and effects of ERB on AIPA processes were causally examined. The results indicated patterns of adopted ERB and AIPA processes differed depending on the level of recognition of enacted SS. However, there were methodological limits in examination of the effects of ERB conducted by injured athletes themselves on reception of SS, with considering their interacting processes. Such limits also existed in examination of correlations among the recovery level of PSRF and SS as well as ERB. Though the recovery level of emotional stability was shown rather low in both high and low SS groups on the measurement scale, it was higher in the low SS group, compared to the high SS group. This result might not have reflected the effect of the recognition level of enacted SS. The level of SS provided to participants maintaining high emotional stability might have been relatively low, and the necessity of using ERB related to emotional expression might be estimated lower. Furthermore, Suzuki and Nakagomi (2013) investigated the content of SS that injured athletes seeked and how support providers changed through the process of rehabilitation. Injured athletes' main complaints are supposed to change according to rehabilitation stages, and SS providers' ways of dealing with athletes' emotions depending on the stage might affect AIPA processes. In the future, rehabilitation for injured athletes should be examined following the time course, and qualitative examination should be conducted on the interactions among the recovery state of PSRF and ERB as well as SS provided by others and effects of these factors on AIPA, taking quality of SS into consideration.

\section{Acknowledgements}

This study was supported by Grant-in-Aid for Scientific Research from Japan Society for the Promotion of Science KAKENHI Grant Number 16K01687.

\section{References}

Cohen, S., Kessler, R. C., \& Gordon, L. U. (1995). Strategies for Measuring Stress in Psychiatric and Physical Disorders. In S. Cohen, R. C. Kessler, \& L. U. Gordon (Eds.), Measuring Stress (pp. 3-28). New York: Oxford University Press.

Freeman, P., Coffee, P., Moll, T., Rees, T., \& Sammy, N. (2014). The ARSQ: The Athletes' Received Support Questionnaire. Journal of Sport \& Exercise Psychology, 36, 189-202. https://doi.org/10.1123/jsep.2013-0080

Fujii, H. (2000). An Approach to Mind of Injured Athletes. Sportsmedicine Quarterly, 27, 59-63.

Gross, J. J., \& John, O. P. (2003). Individual Differences in Two Emotion Regulation Processes: Implications for Affect, Relationships, and Well-Being. Journal of Personality and Social Psychology, 85, 348-362. https://doi.org/10.1037/0022-3514.85.2.348

Iwamitsu, Y., Shimoda, K., Abe, H., Tani, T., Kodama, M., \& Okawa, M. (2003). Differ- 
ences in Emotional Distress between Breast Tumor Patients with Emotional Inhibition and Those with Emotional Expression. Psychiatry and Clinical Neuroscience, 57, 289294. https://doi.org/10.1046/j.1440-1819.2003.01119.x

Kashimura, M., \& Iwamitsu, Y. (2007). Reliability and Validity of the Newly Developed Emotional Suppression Tendency Scale. The Japanese Journal of Health Psychology, 20, 30-41. https://doi.org/10.11560/jahp.20.2_30

Katsumata, T. (1995). Concept and Structure of Time Perspective. Memoirs of the Faculty of Education, Kumamoto University, 44, 307-318.

Kennedy-Moore, E., \& Watson, J. C. (2001). How and When Does Emotional Expression Help? Review of General Psychology, 5, 187-212. https://doi.org/10.1037/1089-2680.5.3.187

Kimura, H. (2004). The Effects of Suppressing Unwanted Thoughts and Replacement Strategy. The Japanese Journal of Educational Psychology, 52, 115-126. https://doi.org/10.5926/jjep1953.52.2_115

Mankad, A., \& Gordon, S. (2010). Psycholinguistic Changes in Athletes' Grief Response to Injury after Written Emotional Disclosure. Journal of Sport Rehabilitation, 19, 328342. https://doi.org/10.1123/jsr.19.3.328

Mankad, A., Gordon, S., \& Wallman, K. (2009a). Perceptions of Emotional Climate among Injured Athletes. Journal of Clinical Sports Psychology, 3, 1-14. https://doi.org/10.1123/jcsp.3.1.1

Mankad, A., Gordon, S., \& Wallman, K. (2009b). Psycholinguistic Analysis of Emotional Disclosure: A Case Study in Sport Injury. Journal of Clinical Sport Psychology, 3, 182196. https://doi.org/10.1123/jcsp.3.2.182

Mankad, A., Gordon, S., \& Wallman, K. (2009c). Psycho-Immunological Effect of Written Emotional Disclosure during Long-Term Injury Rehabilitation. Journal of Clinical Sport Psychology, 3, 205-217. https://doi.org/10.1123/jcsp.3.3.205

McDonald, S. A., \& Hardy, C. J. (1990) Affective Response Patterns of the Injured Athlete: An Exploratory Analysis, The Sport Psychologist, 4, 261-274.

https://doi.org/10.1123/tsp.4.3.261

Mitchell, I. D., Rees, T., Evans, L., \& Hardy, L. (2005). The Development of the Social Support Inventory for Injured Athletes. Proceedings of the Association for the Advancement of Applied Sport Psychology, 102.

Nakagomi, S., \& Uemukai, K. (1994). Counseling for Athletes Who Suffered Injuries. Japanese Journal of Sport Science, 13, 3-8.

Nakatani, Y., Iwamitsu, Y., Kuranami, M., Okazaki, S., Yamamoto, K., Watanabe, M., \& Miyaoka, K. (2012). Emotional Suppression and Psychological Responses to a Diagnosis of Breast Cancer. The Japanese Journal of Psychology, 83, 126-134.

Nakatani, Y., Iwamitsu, Y., Yamamoto, K., \& Miyaoka, H. (2014). Factors Associated with Psychological Distress in Breast Cancer Patients about 1 Year after Surgery. Proceedings of the 78th Annual Convention of the Japanese Psychological Association Proceedings, 646.

Nozaki, Y. (2013). Effects of Regulation of Emotions in the Self and Others on Changes in Emotional Intelligence during a Term Examination Period. The Japanese Journal of Educational Psychology, 61, 362-373. https://doi.org/10.5926/jjep.61.362

Oikawa. M., \& Oikawa, H. (2013). Suppression, Expression, Rumination and Affective Priming Effect. The Japanese Journal of Social Psychology, 29, 40-46.

Rees, T., Mitchell, I. D., Evans, L., \& Hardy, L. (2010). Stressors, Social Support and Psychological Responses to Sport Injury in High and Low Performance Standard Partici- 
pants. Psychology of Sport \& Exercise, 11, 505-512.

https://doi.org/10.1016/j.psychsport.2010.07.002

Stanton, A. L., Danoff-Burg, S., Cameron, C. L., Bishop, M., Collins, C. A., Kirk, S. B., Sworowski, L. A., \& Twillman, R. (2000). Emotionally Expressive Coping Predicts Psychological and Physical Adjustment to Breast Cancer. Journal of Consulting and Clinical Psychology, 68, 875-882. https://doi.org/10.1037/0022-006X.68.5.875

Suzuki, A., \& Nakagomi, S. (2013). Change of Social Support Sought by Injured Athletes during Rehabilitation. Japanese Journal of Sport Psychology, 40, 139-152. https://doi.org/10.4146/jjspopsy.2013-1129

Suzuki, A., \& Nakagomi, S. (2015). Process of Change an Injured Athlete's Coping Behavior While Receiving Social Support through Counseling. Japanese Journal of Clinical Studies for Mind \& Body, 17, 37-48.

Tatsumi, T. (2003). Research on Injury-Acceptance of Athletes: Its Connection with Athletic Rehabilitation Behavior. Annual Bulletin of Oyasato Institute for the Study of Religion, Tenri University, 9, 47-63.

Tatsumi, T. (2012). Psychological Acceptance to Sports Injury in Japanese University Elite Athletes: Coping Free of Adhesion, Perceived Physical Recovery, and Perceived Personal Growth. International Journal of Psychology, 47, 370.

Tatsumi, T. (2013). Development of Athletic Injury Psychological Acceptance Scale. Journal of Physical Therapy Science, 25, 545-552. https://doi.org/10.1589/jpts.25.545

Tatsumi, T. (2014). Relationship between Adaptation after Returning to Competition and Psycho-Behavioral Attitudes during Injury Rehabilitation. Journal of Physical Therapy Science, 26, 1813-1823. https://doi.org/10.1589/jpts.26.1813

Tatsumi, T., \& Nakagomi, S. (1999). A Study on Psychological Acceptance to Injury in Athletes: Analysis from the Viewpoint of Athletic-Rehabilitation Behavior. Japanese Journal of Sport Psychology, 26, 46-57.

Tatsumi, T., \& Takenouchi, T. (2014). Causal Relationships between the Psychological Acceptance Process of Athletic Injury and Athletic Rehabilitation Behavior. Journal of Physical Therapy Science, 26, 1247-1257. https://doi.org/10.1589/jpts.26.1247

Tatsumi, T., Fukumoto, T., \& Bai, D. (2015). Psychological Adjustment to Athletic Injuries. Bulletin of Kio University, 12, 19-26.

Tofighi, D., \& MacKinnon, D. P. (2011). RMediation: An R Package for Mediation Analysis Confidence Intervals. Behavior Research Methods, 43, 692-700. https://doi.org/10.3758/s13428-011-0076-X

Uemukai, K. (1992). Affective Responses and the Changes in Athletes Due to Sport Injury. MS. Thesis, Tsukuba: Tsukuba University.

Uemukai, K. (1993). Affective Responses and the Changes in Athletes due to Injury. Proceedings of the VIII World Congress of Sport Psychology, 500-503.

Wegner, D. M. (1994). Ironic Processes of Mental Control. Psychological Review, 101, 3452. https://doi.org/10.1037/0033-295X.101.1.34

Wegner, D. M., Erber, R., \&Zanakos, S. (1993). Ironic Processes in the Mental Control of Mood and Mood-Related Thought. Journal of Personality and Social Psychology, 65, 1093-1104. https://doi.org/10.1037/0022-3514.65.6.1093

Yoshizu, J. (2014). Mediating Effect of Communication Skill on the Relationship between Emotion Suppression and Well-Being. Proceedings of the 78th Annual Convention of the Japanese Psychological Association.

Yoshizu, J., Sekiguchi, R., \&Amemiya, T. (2013). Development of a Japanese Version of Emotion Regulation Questionnaire. Japanese Journal of Research on Emotions, 20, 5662. https://doi.org/10.4092/jsre.20.56 
Submit or recommend next manuscript to SCIRP and we will provide best service for you:

Accepting pre-submission inquiries through Email, Facebook, LinkedIn, Twitter, etc. A wide selection of journals (inclusive of 9 subjects, more than 200 journals)

Providing 24-hour high-quality service

User-friendly online submission system

Fair and swift peer-review system

Efficient typesetting and proofreading procedure

Display of the result of downloads and visits, as well as the number of cited articles Maximum dissemination of your research work

Submit your manuscript at: http://papersubmission.scirp.org/

Or contact psych@scirp.org 